Bull. Mater. Sci., Vol. 2, Number 2, May 1980, pp. 139-144. (C) Printed in India.

\title{
Evidence of edge and screw dislocations in gypsum single crystals
}

\author{
K S RAJU \\ Physics Department, Madras University Post-Graduate Extension Centre, \\ Coimbatore 641 041, India
}

MS received 11 March 1980; revised 26 April 1980

\begin{abstract}
A row of closely packed equispaced etch pits are observed on etching (010) basal cleavages of gypsum with analar grade nitric acid and $0.1 \mathrm{~N}$ potassium hydroxide solution. It is established that these rows of pits are true to the whole bulk of the crystal, by successive etching and etching of match pairs, thin flakes as well as using different etchants. These rows of pits reveal low angle grain boundaries consisting of equispaced edge dislocations in the crystal. Irregular arrays of etch pits observed exactly correspond to its match face. Studying these rows on higher resolution, it is observed that they may reveal tilt or twist boundaries consisting of both edge and screw dislocations, probably created due to the impingement of the mis-oriented guest crystal causing stresses to the growing crystal. The implications are discussed.
\end{abstract}

Keywords. Chemical etching; low angle grain boundaries; tilt and twist boundaries; spiral etch pits; gypsum single crystal.

\section{Introduction}

Burgers (1940) and Bragg (1940) showed that low angle grain boundaries could be formed by the arrays of edge dislocations. The dislocation model of grain boundaries was confirmed by Vogel and his co-workers (Vogel et al 1953; Vogel 1955), who thoroughly checked the relationship $\theta=b / d$ and established a oneto-one correspondence between etch pits and dislocations. They compared the measured spacing $d$ of etch pits in a tilt boundary in germanium with the orientation change $\theta$, across the boundary. The angle $\theta$ was also measured by the $x$-ray method and the measured spacings agreed with those calculated from the dislocation model of a grain boundary.

The preferential growth along tilt and twist boundaries in hematite crystal was reported by Sunagawa (1968). The irregular lines on (0001) face created due to the impingement of the mis-oriented guest crystal causing stresses to the growing crystal have been observed as tilt and twist boundaries consisting of arrays of edge and screw dislocations respectively. Along these irregular arrays, tonguelike triangular terraces (edge dislocations) and spirals (screw dislocations) are 
shown to be present. Array of minute hills has also been observed on (1011) face of hametite crystal, each of them being a growth spiral.

Patel and Raju (1966) observed spiral depressions on the matched fracture rhombohedral faces of synthetic quartz which are points of emergence of screw dislocations in the crystal.

On etching gypsum cleavages in various etchants, linear rows of closely spaced etch pits and irregular lines of etch pits were obtained, which closely resemble to those observed in germanium and silicon (Vogel et al 1953) and in hematite (Sunagawa 1968) respectively. It was considered worthwhile to investigate if the rows and irregular lines of pits observed revealed the sites of array of dislocations, in which case, they might represent tilt and twist boundaries.

\section{Observations}

\subsection{Reliability of the etchant}

Analar grade nitric acid is the most reliable etchant to reveal the sites of dislocations in gypsum. This could be established from the following experiments :

(i) On successively etching a cleavage for different periods, the etch pits (isolated and as well as those in rows) become larger in size and deeper, depending directly on the duration of etching.

(ii) On etching a pair of matehed cleavage faces under the identical conditions, a perfect one-to-one correspondence of etch pits is established.

(iii) Arrays of etch pits having exact correlation on the opposite sides of the etched crystal flake, and on etching a very thin flake for a prolonged time, holes having the shape of etch pits are observed at a few isolated sites, the array tending to a groove, and,

(iv) From the top of the crystal etched in nitric acid revealing a row of etch pits, a small flake is cleaved out and on etching the fresh face with $0.1 \mathrm{~N}$ potassium hydroxide solution, the row of etch pits re-appears at the same position.

Potassium hydroxide $(0.1 \mathrm{~N})$ solution is also a reliable dislocation etchant but the crystal $\left(\mathrm{CaSO}_{4} \cdot 2 \mathrm{H}_{2} \mathrm{O}\right)$ face on prolonged etching becomes unclean due to the formation of white precipitate of calcium hydroxide.

\subsection{Grain boundaries}

2.2a. Etching of isolated (010) cleavages. Figure 1 represents the etch pattern produced by etching a cleavage face in analar grade nitric acid for 2 min. The row of etch pits is clearly seen and assuming that it reveals a grain boundary, the spacing of the pits was measured and from the lattice constant of the crystal, the angle between the two grains was computed to be $1 \mathrm{~min}$. In the present investigations, the spacing between the pits was calculated by counting the number of pits in $5 \mathrm{~cm}$ length at a magnification of about $\times 1000$. Since the tilt is very small, the orientation of rhombohedral pits on the two sides of the boundary appears to be the same. Figure 2 clearly shows the isolated pits situated in a row.

2.26. Successive etching of isolated cleavages. A cleaved face, revealing the arrays of etch pits described above, was successively etched for 3 and 9 min respectively. 

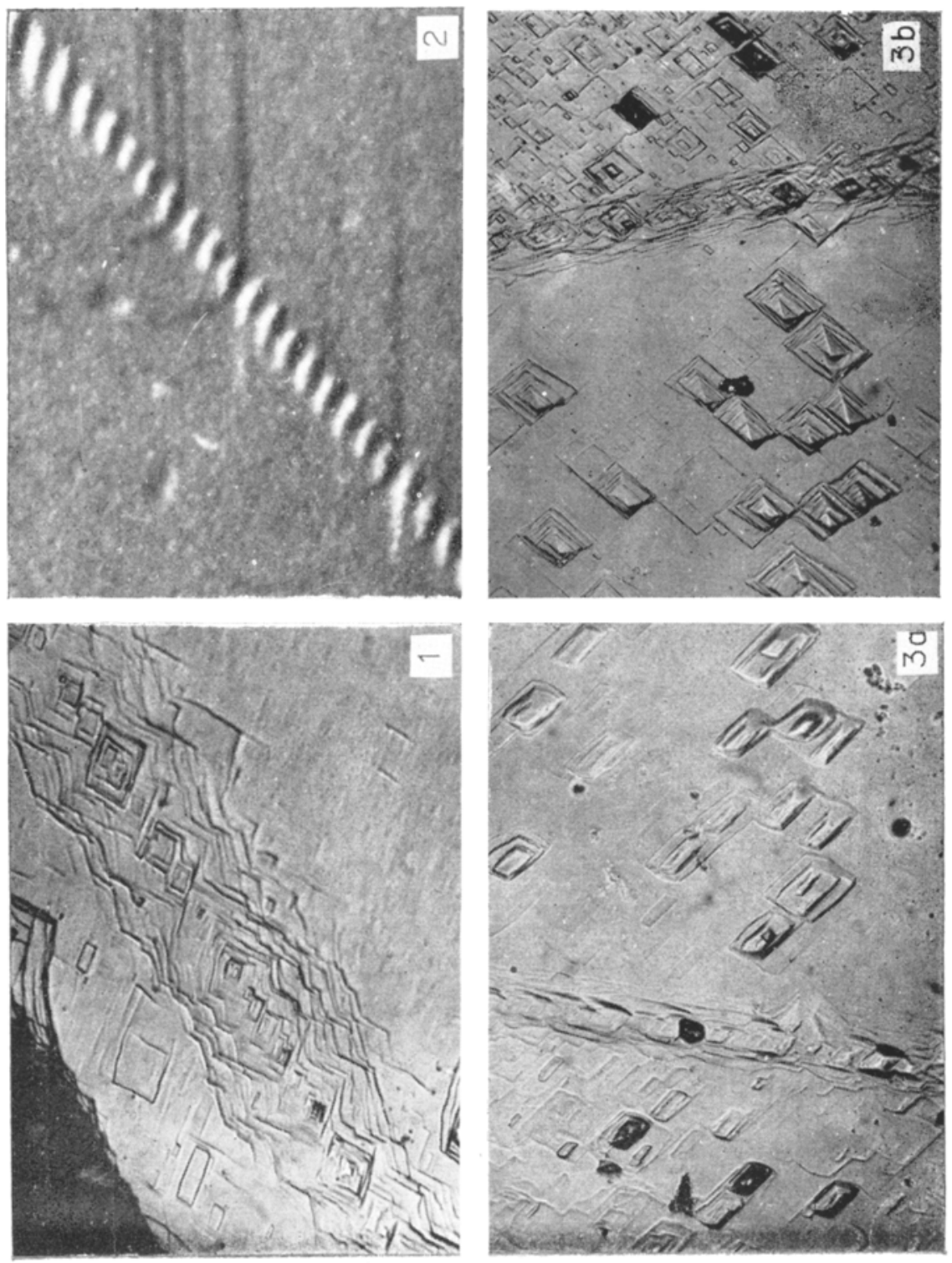

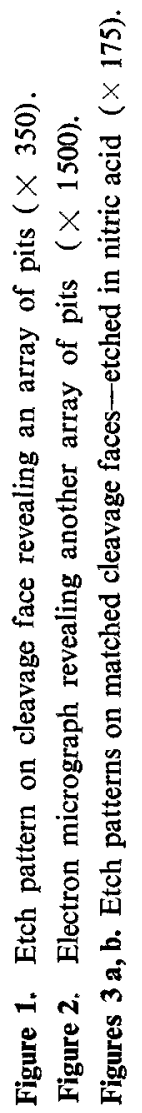



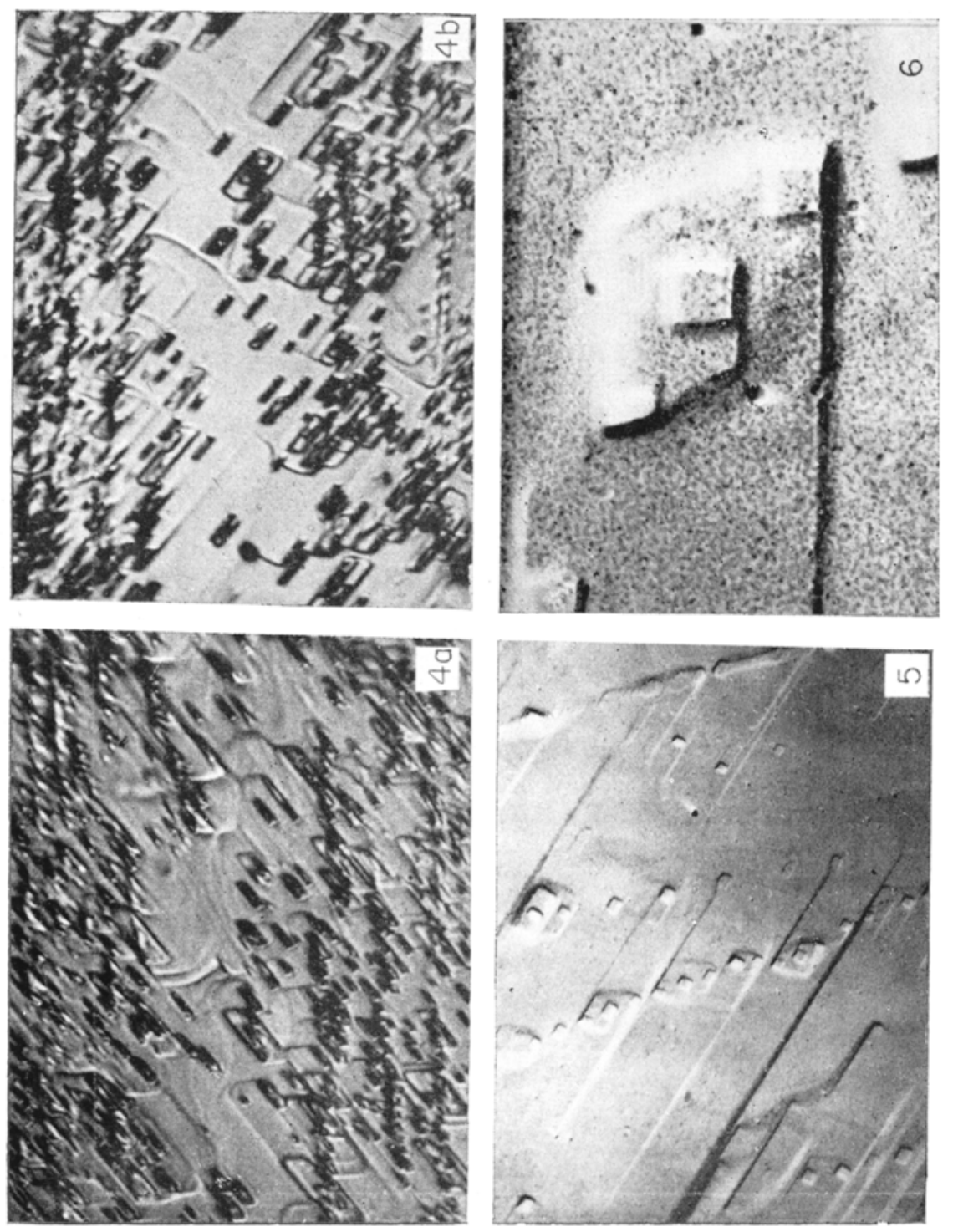

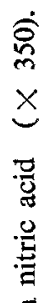

믐

형

is $x$

焉

离空

क 4

卷志

导

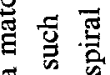

대

.

岁司

赵 돌

$\Rightarrow$ to

密 鄫

总

동

$\therefore \frac{\mathrm{U}}{\mathrm{u}}$

$\checkmark$ vi

参吉 
At each stage, the spacing between the pits was calculated and this was numerically the same within the experimental error.

2.2c. Etching of matched cleavage faces and flakes, To determine whether these rows represent low angle grain boundaries, a pair of matched cleavage faces was etched in nitric acid. Figures $3(\mathrm{a})$ and $3(\mathrm{~b})$ illustrate the etch patterns thus produced. Exastly similar row of pits is produced along with the isolated pits on the matched faces having perfect correlation. The average distance between the two pits is the same in both the matched faces.

Two parallel arrays of pits exactly corresponding to both the opposite sides of the flake ( $2 \mathrm{~mm}$ thick) are observed on etching in nitric acid for $4 \mathrm{~min}$. The average spacing of the pits in the row was practically the same within the experimental error.

2.2d. Etching with different etchants. A gypsum flake revealing a row of etch pits is etched in nitric acid. A thin flake (of $0.023 \mathrm{~mm}$ thickness) is removed from the top of the above etched crystal by cleaving and the fresh face is etched in $0.1 \mathrm{~N}$ potassium hydroxide solution. The spacing as measured between the consecutive pits in the row of etch pits formed by two different etchants is the same.

\subsection{Irregular arrays of etch pits}

Figures 4 (a) and 4 (b) show the etch patterns on the match faces of gypsum revealing the irregular arrays of etch pits. A one-to-one correspondence of irregular arrays can be established from these figures.

The irregular arrays of etch pits was studied at a higher resolution. Figure 5 reveals clearly two such arrays of pits shown in figures 4 (a) and 4 (b). It is interesting to note that most of the etch pits along such arrays appear as terraced pits. But an examination of some of these pits at a higher magnification and greater resolution shows that these are spiral pits. Figure 6 shows the spiral nature of etch pits almost linearly arranged.

\section{Discussion}

From the observations of successive etching, etching of match pairs and flakes, it is clear that the rows of etch pits observed are dislocation etch pits. The uniformity in the average spacing of the consecutive pits, throughout, supports the view, that the array of etch pits reveals the array of edge dislocations, forming the low angle grain boundary.

Exact correspondence of the etch patterns obtained by etching in different etchants, on match faces as well as on opposite sides of a flake suggests that the low angle of mis-orientation is true even to the interior of the crystal. Etch pit data for determining the angle of mis-orientation suggests that these rows are low angle grain boundaries in the crystal.

A one-to-one correspondence of the irregular arrays of etch pits on the matched pair suggests that they reveal the sites of dislocations. Some of them which are spirals having rhombohedral shape may reveal the sites of screw dislocations 
present on general grain boundaries having both edge and screw dislocations. The irregular lines of etch pits discussed above might have been formed due to the impingement of the mis-oriented guest crystal causing stresses in the growing crystal (Sunagawa 1968).

\section{Acknowledgements}

The author thanks Prof. A R Patel, Department of Physics, Sardar Patel University, Vallabh Vidyanagar, for his encouragement.

\section{References}

Bragg W L 1940 Proc. Phys. Soc. 5254

Burgers W L 1940 Proc. Phys. Soc. 89468

Patel A R and Raju K S 1966 Acta Crystallogr. 21190

Sunagawa I 1968 Mineral Mag. 36625

Vogel F L 1955 Acta Met. 3245

Vogel F L, Pfann W E, Corey H F and Thomas E E 1953 Phys. Rev. 90489 